\title{
Isthmus-Based Parallel and Asymmetric 3D Thinning Algorithms ${ }^{\star}$
}

\author{
Michel Couprie and Gilles Bertrand \\ Université Paris-Est, LIGM, Équipe A3SI, ESIEE Paris, France \\ \{michel.couprie, gilles bertrand\}@esiee.fr
}

\begin{abstract}
Critical kernels constitute a general framework settled in the context of abstract complexes for the study of parallel thinning in any dimension. We take advantage of the properties of this framework, to propose a generic thinning scheme for obtaining "thin" skeletons from objects made of voxels. From this scheme, we derive algorithms that produce curvilinear or surface skeletons, based on the notion of $1 \mathrm{D}$ or $2 \mathrm{D}$ isthmus.
\end{abstract}

\section{Introduction}

When dealing with skeletons, one has to face two main problems: topology preservation, and preservation of meaningful geometrical features. Here, we are interested in the skeletonization of objects that are made of voxels (unit cubes) in a regular 3D grid, i.e., in a binary 3D image. In this context, topology preservation is usually obtained through the iteration of thinning steps, provided that each step does not alter the topological characteristics. In sequential thinning algorithms, each step consists of detecting and choosing a so-called simple voxel, that may be characterized locally (see [12]), and removing it. Such a process usually involves many arbitrary choices, and the final result may depend, sometimes heavily, on any of these choices. This is why parallel thinning algorithms are generally preferred to sequential ones. However, removing a set of simple voxels at each thinning step, in parallel, may alter topology. The framework of critical kernels, introduced by one of the authors in [3, provides a condition under which we have the guarantee that a subset of voxels can be removed without changing topology. This condition is, to our knowledge, the most general one among the related works. Furthermore, critical kernels indeed provide a method to design new parallel thinning algorithms, in which the property of topology preservation is built-in, and in which any kind of constraint may be imposed (see [45]).

Among the different parallel thinning algorithms that have been proposed in the literature, we can distinguish symmetric from asymmetric algorithms. Symmetric algorithms (see e.g. 6778]) (also known as fully parallel algorithms) produce skeletons that are invariant under 90 degrees rotations. They consist of the iteration of thinning steps that are made of 1) the identification and selection

\footnotetext{
* This work has been partially supported by the "ANR-2010-BLAN-0205 KIDICO" project.
} 
of a set of voxels that satisfy certain conditions, independently of orientation or position in space, and 2) the removal, in parallel, of all selected voxels from the object. Symmetric algorithms, on the positive side, produce a result that is uniquely defined: no arbitrary choice is needed. On the negative side, they generally produce thick skeletons, see Fig. 1 .

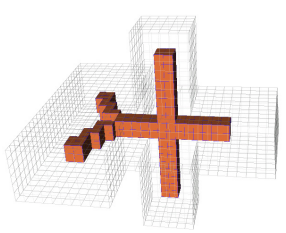

(a)

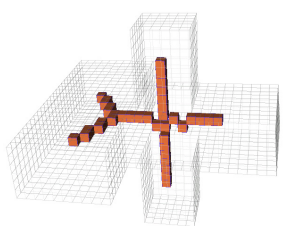

(b)

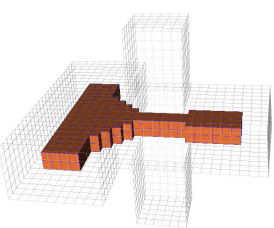

(c)

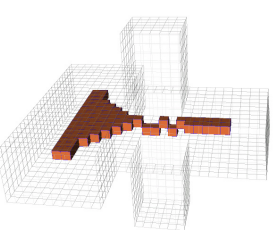

(d)

Fig. 1. Different types of skeletons. (a): Curvilinear skeleton, symmetric. (b): Curvilinear skeleton, asymmetric. (c): Surface skeleton, symmetric. (d): Surface skeleton, asymmetric.

Asymmetric skeletons, on the opposite, are preferred when thinner skeletons are required. The price to pay is a certain amount of arbitrary choices to be made. In all existing asymmetric parallel thinning algorithms, each thinning step is divided into a certain number of substeps. In the so-called directional algorithms (see e.g. 91011 ), each substep is devoted to the detection and the deletion of voxels belonging to one "side" of the object: all the voxels considered during the substep have, for example, their south neighbor inside the object and their north neighbor outside the object. The order in which these directional substeps are executed is set beforehand, arbitrarily. Subgrid (or subfield) algorithms (see e.g. 1213 ) form the second category of asymmetric parallel thinning algorithms. There, each substep is devoted to the detection and the deletion of voxels that belong to a certain subgrid, for example, all voxels that have even coordinates. Considered subgrids must form a partition of the grid. Again, the order in which subgrids are considered is arbitrary.

Subgrid algorithms are not often used in practice because they produce artifacts, that is, waving skeleton branches where the original object is smooth or straight. Directional algorithms are the most popular ones. Most of them are implemented through sets of masks, one per substep. A set of masks is used to characterize voxels that must be kept during a given substep, in order to 1) preserve topology, and 2) prevent curves or surfaces to disappear. Thus, topological conditions and geometrical conditions cannot be easily distinguished, and the slightest modification of any mask involves the need to make a new proof of the topological correctness.

Our approach is radically different. Instead of considering single voxels, we consider cliques. A clique is a set of mutually adjacent voxels. Then, we identify 
the critical kernel of the object, according to some definitions, which is a union of cliques. The main theorem of the critical kernels framework [35] states that we can remove in parallel any subset of the object, provided that we keep at least one voxel of every clique that constitutes the critical kernel, and this guarantees topology preservation. Here, as we try to obtain thin skeletons, our goal is to keep, whenever possible, exactly one voxel in every such clique. This leads us to propose a generic parallel asymmetric thinning scheme, that may be enriched by adding any sort of geometrical constraint. For example, we define the notions of $1 \mathrm{D}$ and $2 \mathrm{D}$ isthmuses. A $1 \mathrm{D}$ (resp. 2D) isthmus is a voxel that is "locally like a piece of curve" (resp. surface). From our generic scheme, we easily derive, by adding the constraint to preserve isthmuses, specific algorithms that produce curvilinear or surface skeletons.

\section{Voxel Complexes}

In this section, we give some basic definitions for voxel complexes, see also [141].

Let $\mathbb{Z}$ be the set of integers. We consider the families of sets $\mathbb{F}_{0}^{1}, \mathbb{F}_{1}^{1}$, such that $\mathbb{F}_{0}^{1}=\{\{a\} \mid a \in \mathbb{Z}\}, \mathbb{F}_{1}^{1}=\{\{a, a+1\} \mid a \in \mathbb{Z}\}$. A subset $f$ of $\mathbb{Z}^{n}, n \geq 2$, that is the Cartesian product of exactly $d$ elements of $\mathbb{F}_{1}^{1}$ and $(n-d)$ elements of $\mathbb{F}_{0}^{1}$ is called a face or an $d$-face of $\mathbb{Z}^{n}, d$ is the dimension of $f$. In the illustrations of this paper except Fig. 6] a 3-face (resp. 2-face, 1-face, 0-face) is depicted by a cube (resp. square, segment, dot), see e.g. Fig. 4,

A 3 -face of $\mathbb{Z}^{3}$ is also called a voxel. A finite set that is composed solely of voxels is called a (voxel) complex (see Fig. 2). We denote by $\mathbb{V}^{3}$ the collection of all voxel complexes.

We say that two voxels $x, y$ are adjacent if $x \cap y \neq \emptyset$. We write $\mathcal{N}(x)$ for the set of all voxels that are adjacent to a voxel $x, \mathcal{N}(x)$ is the neighborhood of $x$. Note that, for each voxel $x$, we have $x \in \mathcal{N}(x)$. We set $\mathcal{N}^{*}(x)=\mathcal{N}(x) \backslash\{x\}$.

Let $d \in\{0,1,2\}$. We say that two voxels $x, y$ are $d$-neighbors if $x \cap y$ is a $d$-face. Thus, two distinct voxels $x$ and $y$ are adjacent if and only if they are $d$-neighbors for some $d \in\{0,1,2\}$.

Let $X \in \mathbb{V}^{3}$. We say that $X$ is connected if, for any $x, y \in X$, there exists a sequence $\left\langle x_{0}, \ldots, x_{k}\right\rangle$ of voxels in $X$ such that $x_{0}=x, x_{k}=y$, and $x_{i}$ is adjacent to $x_{i-1}, i=1, \ldots, k$.

\section{Simple Voxels}

Intuitively a voxel $x$ of a complex $X$ is called a simple voxel if its removal from $X$ "does not change the topology of $X$ ". This notion may be formalized with the help of the following recursive definition introduced in [5], see also [15]16] for other recursive approaches for simplicity.

Definition 1. Let $X \in \mathbb{V}^{3}$.

We say that $X$ is reducible if either:

i) $X$ is composed of a single voxel; or

ii) there exists $x \in X$ such that $\mathcal{N}^{*}(x) \cap X$ is reducible and $X \backslash\{x\}$ is reducible. 


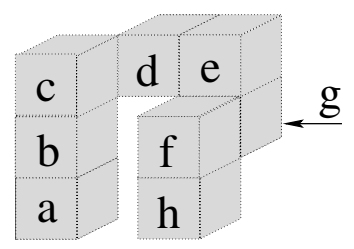

(a)

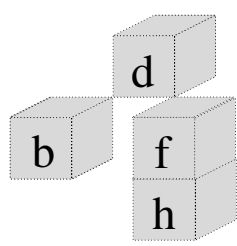

(b)

Fig. 2. (a) A complex $X$ which is made of 8 voxels, (b) A complex $Y \subseteq X$, which is a thinning of $X$

Definition 2. Let $X \in \mathbb{V}^{3}$. A voxel $x \in X$ is simple for $X$ if $\mathcal{N}^{*}(x) \cap X$ is reducible. If $x \in X$ is simple for $X$, we say that $X \backslash\{x\}$ is an elementary thinning of $X$.

Thus, a complex $X \in \mathbb{V}^{3}$ is reducible if and only if it is possible to reduce $X$ to a single voxel by iteratively removing simple voxels. Observe that a reducible complex is necessarily non-empty and connected.

In Fig. 2 (a), the voxel $a$ is simple for $X\left(\mathcal{N}^{*}(a) \cap X\right.$ is made of a single voxel), the voxel $d$ is not simple for $X\left(\mathcal{N}^{*}(d) \cap X\right.$ is not connected), the voxel $h$ is simple for $X\left(\mathcal{N}^{*}(h) \cap X\right.$ is made of two voxels that are 2-neighbors and is reducible).

In [5], it was shown that the above definition of a simple voxel is equivalent to classical characterizations based on connectivity properties of the voxel's neighborhood [17/18|19|20/2. An equivalence was also established with a definition based on the operation of collapse [21], this operation is a discrete analogue of a continuous deformation (a homotopy), see also 15/312.

The notion of a simple voxel allows one to define thinnings of a complex, see an illustration Fig. 2 (b).

Let $X, Y \in \mathbb{V}^{3}$. We say that $Y$ is a thinning of $X$ or that $X$ is reducible to $Y$, if there exists a sequence $\left\langle X_{0}, \ldots, X_{k}\right\rangle$ such that $X_{0}=X, X_{k}=Y$, and $X_{i}$ is an elementary thinning of $X_{i-1}, i=1, \ldots, k$.

Thus, a complex $X$ is reducible if and only if it is reducible to a single voxel.

\section{Critical Kernels}

Let $X$ be a complex in $\mathbb{V}^{3}$. It is well known that, if we remove simultaneously (in parallel) simple voxels from $X$, we may "change the topology" of the original object $X$. For example, the two voxels $f$ and $g$ are simple for the object $X$ depicted Fig. 2 (a). Nevertheless $X \backslash\{f, g\}$ has two connected components whereas $X$ is connected.

In this section, we recall a framework for thinning in parallel discrete objects with the warranty that we do not alter the topology of these objects [3/4/5. This method is valid for complexes of arbitrary dimension.

Let $d \in\{0,1,2,3\}$ and let $C \in \mathbb{V}^{3}$. We say that $C$ is a $d$-clique or a clique if $\cap\{x \in C\}$ is a $d$-face. If $C$ is a $d$-clique, $d$ is the rank of $C$. 
If $C$ is made of solely two distinct voxels $x$ and $y$, we note that $C$ is a $d$-clique if and only if $x$ and $y$ are $d$-neighbors, with $d \in\{0,1,2\}$.

Let $X \in \mathbb{V}^{3}$ and let $C \subseteq X$ be a clique. We say that $C$ is essential for $X$ if we have $C=D$ whenever $D$ is a clique such that:

i) $C \subseteq D \subseteq X$; and

ii) $\cap\{x \in \bar{C}\}=\cap\{x \in D\}$.

Observe that any complex $C$ that is made of a single voxel is a clique (a 3 -clique). Furthermore any voxel of a complex $X$ constitutes a clique that is essential for $X$.

In Fig. 2 (a), $\{f, g\}$ is a 2-clique that is essential for $X,\{b, d\}$ is a 0 -clique that is not essential for $X,\{b, c, d\}$ is a 0 -clique essential for $X,\{e, f, g\}$ is a 1-clique essential for $X$.

Definition 3. Let $S \in \mathbb{V}^{3}$. The $\mathcal{K}$-neighborhood of $S$, written $\mathcal{K}(S)$, is the set made of all voxels that are adjacent to each voxel in $S$. We set $\mathcal{K}^{*}(S)=\mathcal{K}(S) \backslash S$.

We note that we have $\mathcal{K}(S)=\mathcal{N}(x)$ whenever $S$ is made of a single voxel $x$. We also observe that we have $S \subseteq \mathcal{K}(S)$ whenever $S$ is a clique.

Definition 4. Let $X \in \mathbb{V}^{3}$ and let $C$ be a clique that is essential for $X$. We say that the clique $C$ is regular for $X$ if $\mathcal{K}^{*}(C) \cap X$ is reducible. We say that $C$ is critical for $X$ if $C$ is not regular for $X$.

Thus, if $C$ is a clique that is made of a single voxel $x$, then $C$ is regular for $X$ if and only if $x$ is simple for $X$.

In Fig. 2 (a), the cliques $C_{1}=\{b, c, d\}, C_{2}=\{f, g\}$, and $C_{3}=\{f, h\}$ are essential for $X$. We have $\mathcal{K}^{*}\left(C_{1}\right) \cap X=\emptyset, \mathcal{K}^{*}\left(C_{2}\right) \cap X=\{e, h\}$, and $\mathcal{K}^{*}\left(C_{3}\right) \cap X=$ $\{g\}$. Thus, $C_{1}$ and $C_{2}$ are critical for $X$, while $C_{3}$ is regular for $X$.

The following result is a consequence of a general theorem that holds for complexes of arbitrary dimensions [35], see an illustration Fig. 2] (a) and (b) where the complexes $X$ and $Y$ satisfy the condition of Th. 5 .

Theorem 5. Let $X \in \mathbb{V}^{3}$ and let $Y \subseteq X$.

The complex $Y$ is a thinning of $X$ if any clique that is critical for $X$ contains at least one voxel of $Y$.

\section{A Generic 3D Parallel and Asymmetric Thinning Scheme}

Our goal is to define a subset $Y$ of a voxel complex $X$ that is guaranteed to include at least one voxel of each clique that is critical for $X$. By Th. 5, this subset $Y$ will be a thinning of $X$.

Let us consider the complex $X$ depicted Fig. 3 (a). There are precisely three cliques that are critical for $X$ :

- the 0-clique $C_{1}=\{b, c\}$ (we have $\left.\mathcal{K}^{*}\left(C_{1}\right) \cap X=\emptyset\right)$;

- the 2-clique $C_{2}=\{a, b\}$ (we have $\mathcal{K}^{*}\left(C_{2}\right) \cap X=\emptyset$ );

- the 3 -clique $C_{3}=\{b\}$ (the voxel $b$ is not simple). 
Suppose that, in order to build a complex $Y$ that fulfills the condition of Th. 5. we select arbitrarily one voxel of each clique that is critical for $X$. Following such a strategy, we could select $c$ for $C_{1}, a$ for $C_{2}$, and $b$ for $C_{3}$. Thus, we would have $Y=X$, no voxel would be removed from $X$. Now, we observe that the complex $Y^{\prime}=\{b\}$ satisfies the condition of Th. 5. This complex is obtained by considering first the 3-cliques before selecting a voxel in the 2-, 1-, or 0 cliques.

The complex $X$ of Fig. 3 (b) provides another example of such a situation. There are precisely three cliques that are critical for $X$ :

- the 1-clique $C_{1}=\{e, f, g, h\}$ (we have $\left.\mathcal{K}^{*}\left(C_{1}\right) \cap X=\emptyset\right)$;

- the 1-clique $C_{2}=\{e, d, g\}$ (we have $\mathcal{K}^{*}\left(C_{2}\right) \cap X=\emptyset$ );

- the 2-clique $C_{3}=\{e, g\}\left(\mathcal{K}^{*}\left(C_{3}\right) \cap X\right.$ is not connected).

If we select arbitrarily one voxel of each critical clique, we could obtain the complex $Y=\{f, d, g\}$. On the other hand, if we consider the 2-cliques before the 1-cliques, we obtain either $Y^{\prime}=\{e\}$ or $Y^{\prime \prime}=\{g\}$. In both cases the result is better in the sense that we remove more voxels from $X$.

This discussion motivates the introduction of the following 3D asymmetric and parallel thinning scheme AsymThinningScheme(see also [415]). The main features of this scheme are the following:

- Taking into account the observations made through the two previous examples, critical cliques are considered according to their decreasing ranks (step 4). Thus, each iteration is made of four sub-iterations (steps 4-8). Voxels that have been previously selected are stored in a set $Y$ (step 8). At a given sub-iteration, we consider voxels only in critical cliques included in $X \backslash Y$ (step 6).

- Select is a function from $\mathbb{V}^{3}$ to $V^{3}$, the set of all voxels. More precisely, Select associates, to each set $S$ of voxels, a unique voxel $x$ of $S$. We refer to such a function as a selection function. This function allows us to select a voxel in a given critical clique (step 7). A possible choice is to take for $\operatorname{Select}(S)$, the first pixel of $S$ in the lexicographic order of the voxels coordinates.

- In order to compute curvilinear or surface skeletons, we have to keep other voxels than the ones that are necessary for the preservation of the topology of the object $X$. In the scheme, the set $K$ corresponds to a set of features that we want to be preserved by a thinning algorithm (thus, we have $K \subseteq X$ ). This set $K$, called constraint set, is updated dynamically at step $10 . S_{k e l_{X}}$ is a function from $X$ on $\{$ True, False $\}$ that allows us to keep some skeletal voxels of $X, e . g$., some voxels belonging to parts of $X$ that are surfaces or curves. For example, if we want to obtain curvilinear skeletons, a popular solution is to set $\operatorname{Skel}_{X}(x)=$ True whenever $x$ is a so-called end voxel of $X$ : an end voxel is a voxel that has exactly one neighbor inside $X$; see Fig. 7(a) a skeleton obtained in this way. However, this solution is limited and does not permit to obtain surface skeletons. Better propositions for such a function will be introduced in section 6

By construction, at each iteration, the complex $Y$ at step 9 satisfies the condition of Th. 5. Thus, the result of the scheme is a thinning of the original complex $X$. Observe also that, except step 4 , each step of the scheme may be computed in parallel. 


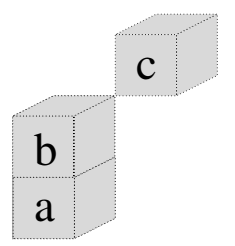

(a)

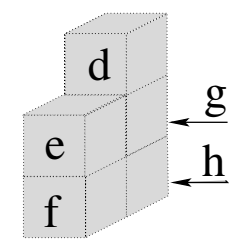

(b)

Fig. 3. Two complexes

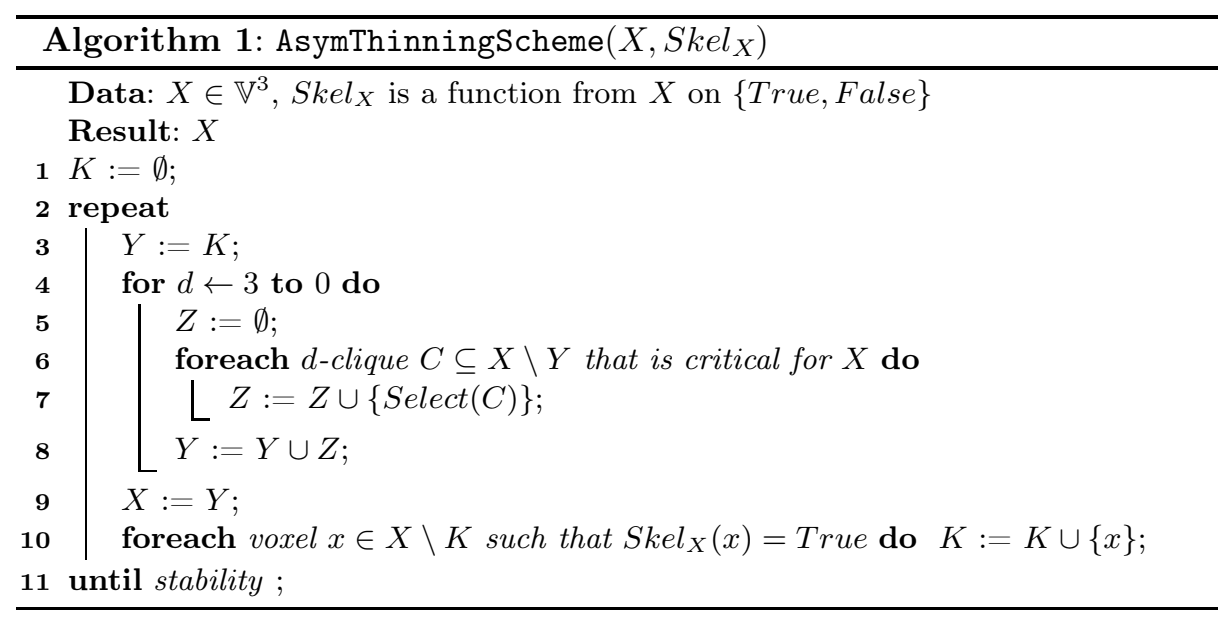

Fig. 4 provides an illustration of the scheme AsymThinningScheme. Let us consider the complex $X$ depicted in (a). We suppose in this example that we do not keep any skeletal voxel, i.e., for any $x \in X$, we set $\operatorname{Skel}_{X}(x)=$ False. The traces of the cliques that are critical for $X$ are represented in (b), the trace of a clique $C$ is the face $f=\cap\{x \in C\}$. Thus, the set of the cliques that are critical for $X$ is precisely composed of six 0-cliques, two 1-cliques, three 2-cliques, and one 3-clique. In (c) the different sub-iterations of the scheme are illustrated (steps 4-8):

- when $d=3$, only one clique is considered, the dark grey voxel is selected whatever the selection function;

- when $d=2$, all the three 2-cliques are considered since none of these cliques contains the above voxel. Voxels that could be selected by a selection function are depicted in medium grey;

- when $d=1$, only one clique is considered, a voxel that could be selected is depicted in light grey;

- when $d=0$, no clique is considered since each of the 0 -cliques contains at least one voxel that has been previously selected.

After these sub-iterations, we obtain the complex depicted in (d). The figures (e) and (f) illustrate the second iteration, at the end of this iteration the complex 


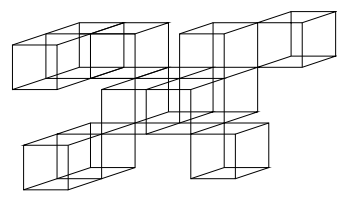

(a)

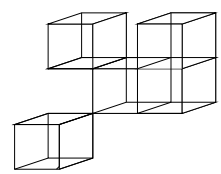

(d)

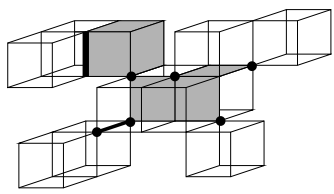

(b)

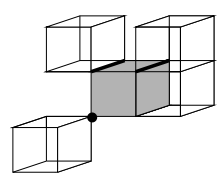

(e)

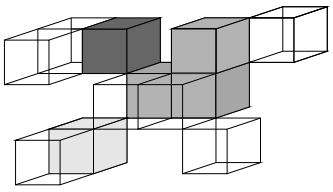

(c)

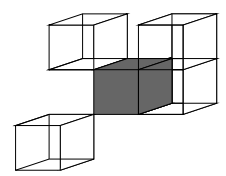

(f)

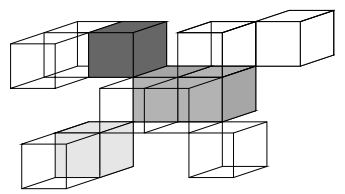

(g)

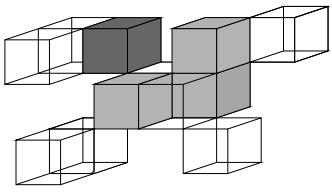

(h)

Fig. 4. (a): A complex $X$ made of precisely 12 voxels. (b): The traces of the cliques that are critical for $X$. (c): Voxels that have been selected by the algorithm. (d): The result $Y$ of the first iteration. (e): The traces of the 4 cliques that are critical for $Y$. (f): The result of the second iteration. $(\mathrm{g})$ and $(\mathrm{h})$ : Two other possible selections at the first iteration.

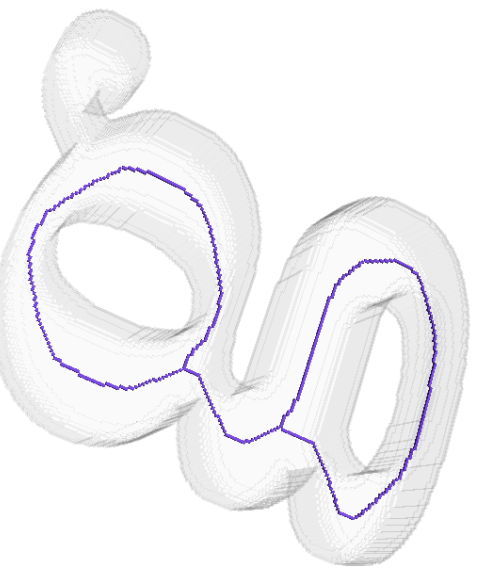

Fig. 5. Ultimate asymmetric skeletons obtained by using AsymThinningScheme 


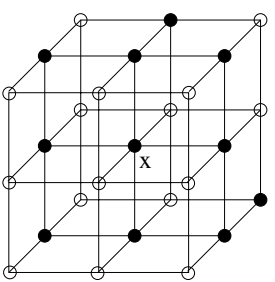

(a)

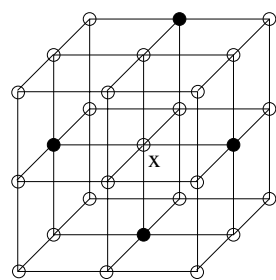

(b)

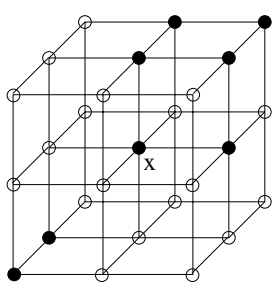

(c)

Fig. 6. In this figure, a voxel is represented by its central point. (a): A voxel $x$ and the set $\mathcal{N}(x) \cap X$ (black points). (b): A set $S$ which is a 1 -surface, $\mathcal{N}^{*}(x) \cap X$ is reducible to $S$, thus $x$ is a 2-isthmus. for $X$. (c): A voxel $x$ and the set $\mathcal{N}(x) \cap X$ (black points). The voxel $x$ is a 1-isthmus for $X$.

is reduced to a single voxel. In (g) and (h) two other possible selections at the first iteration are given.

Of course, the result of the scheme may depend on the choice of the selection function. This is the price to be paid if we try to obtain thin skeletons. For example, some arbitrary choices have to be made for reducing a two voxels wide ribbon to a simple curve.

In the sequel of the paper, we take for $\operatorname{Select}(S)$, the first pixel of $S$ in the lexicographic order of the voxels coordinates.

Fig. [5] shows another illustration, on bigger objects, of AsymThinningScheme. Here also, for any $x \in X$, we have $\operatorname{Skel}_{X}(x)=$ False (no skeletal voxel). The result is called an ultimate asymmetric skeleton.

\section{Isthmus-Based Asymmetric Thinning}

In this section, we show how to use our generic scheme AsymThinningScheme in order to get a procedure that computes either curvilinear or surface skeletons. This thinning procedure preserves a constraint set $K$ that is made of "isthmuses".

Intuitively, a voxel $x$ of an object $X$ is said to be a 1 -isthmus (resp. a 2isthmus) if the neighborhood of $x$ corresponds - up to a thinning - to the one of a point belonging to a curve (resp. a surface) [5].

We say that $X \in \mathbb{V}^{3}$ is a 0 -surface if $X$ is precisely made of two voxels $x$ and $y$ such that $x \cap y=\emptyset$.

We say that $X \in \mathbb{V}^{3}$ is a 1-surface (or a simple closed curve) if:

i) $X$ is connected; and ii) For each $x \in X, \mathcal{N}^{*}(x) \cap X$ is a 0 -surface.

Definition 6. Let $X \in \mathbb{V}^{3}$, let $x \in X$.

We say that $x$ is a 1 -isthmus for $X$ if $\mathcal{N}^{*}(x) \cap X$ is reducible to a 0 -surface. We say that $x$ is a 2 -isthmus for $X$ if $\mathcal{N}^{*}(x) \cap X$ is reducible to a 1 -surface. We say that $x$ is a $2^{+}$-isthmus for $X$ if $x$ is a 1 -isthmus or a 2 -isthmus for $X$. 


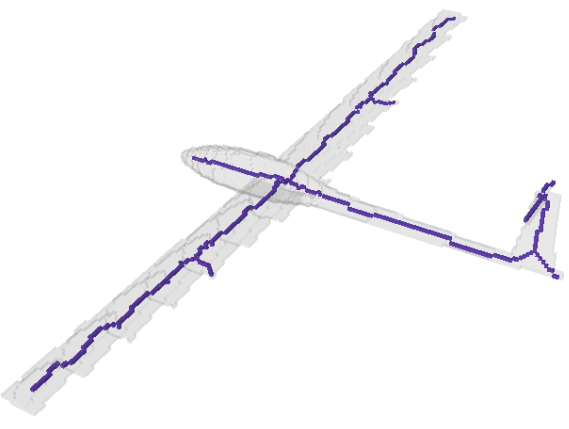

(a)

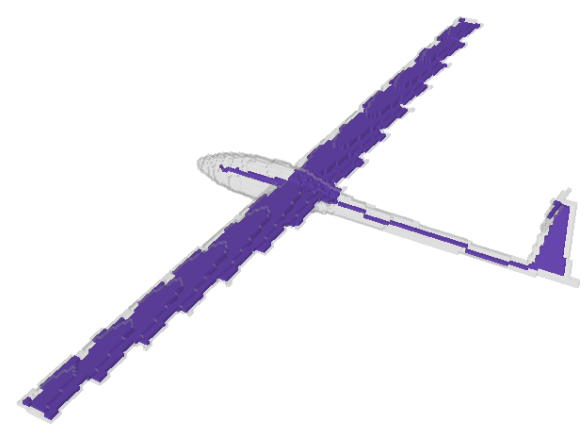

(c)

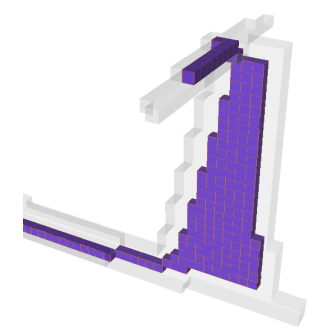

(e)

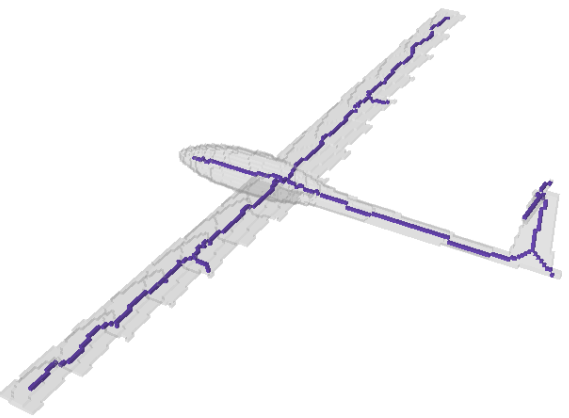

(b)

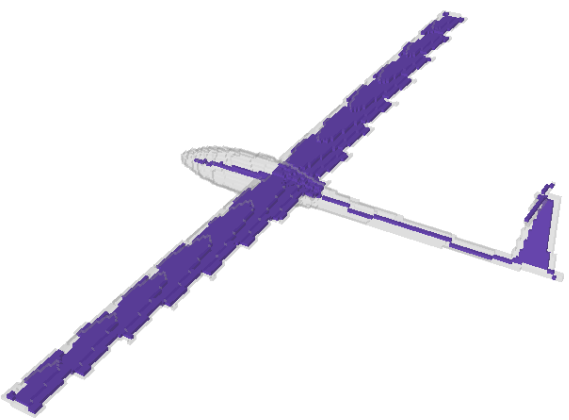

(d)

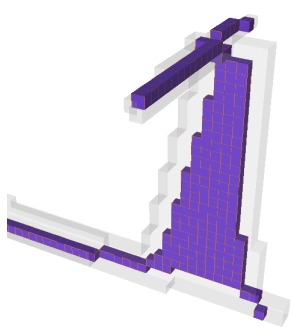

(f)

Fig. 7. Asymmetric skeletons obtained by using AsymThinningScheme. (a): the function $S k e l_{X}$ is based on end voxels. (b,c,d): the function $S k e l_{X}$ is based on $k$-isthmuses, with $k=1,2$ and $2^{+}$respectively. $(\mathrm{e}, \mathrm{f})$ : detail of $(\mathrm{c}, \mathrm{d})$ respectively. 
Our aim is to thin an object, while preserving a constraint set $K$ that is made of voxels that are detected as $k$-isthmuses during the thinning process. We obtain curvilinear skeletons with $k=1$, surface skeletons with $k=2$, and surface/curvilinear skeletons with $k=2^{+}$. These three kinds of skeletons may be obtained by using AsymThinningScheme, with the function Skel $_{X}$ defined as follows:

$$
\text { Skel }_{X}(x)=\left\{\begin{array}{l}
\text { True if } x \text { is a } k \text {-isthmus, } \\
\text { False otherwise, }
\end{array}\right.
$$

with $k \in\left\{1,2,2^{+}\right\}$.

Observe there is the possibility that a voxel belongs to a $k$-isthmus at a given step of the algorithm, but not at further steps. This is why previously detected isthmuses are stored (see line 10 of AsymThinningScheme).

In Fig. 7(b-f), we show a curvilinear skeleton, a surface skeleton and a surface/curvilinear skeleton obtained by our method from the same object.

\section{Conclusion}

We introduced an original generic scheme for asymmetric parallel topologypreserving thinning of 3D objects made of voxels, in the framework of critical kernels. We saw that from this scheme, one can easily derive several thinning operators having specific behaviours, simply by changing the definition of skeletal points. In particular, we showed that ultimate, curvilinear, surface, and surface/curvilinear skeletons can be obtained, based on the notion of $1 \mathrm{D} / 2 \mathrm{D}$ isthmuses.

A key point, in the implementation of the algorithms proposed in this paper, is the detection of critical cliques and isthmus voxels. In [5], we showed that it is possible to detect critical cliques thanks to a set of masks, in linear time. We also showed that the configurations of $1 \mathrm{D}$ and $2 \mathrm{D}$ isthmuses may be pre-computed by a linear-time algorithm and stored in lookup tables. Finally, based on a breadthfirst strategy, the whole method can be implemented to run in $O(n)$ time, where $n$ is the number of voxels of the input $3 \mathrm{D}$ image.

In an extended paper, in preparation, we will show how to deal with the robustness to noise issue thanks to the notion of isthmus persistence. We will also compare our method with all existing asymmetric parallel skeletonization algorithms acting in the 3D cubic grid.

\section{References}

1. Kong, T.Y., Rosenfeld, A.: Digital topology: introduction and survey. Comp. Vision, Graphics and Image Proc. 48, 357-393 (1989)

2. Couprie, M., Bertrand, G.: New characterizations of simple points in 2D, 3D and $4 \mathrm{D}$ discrete spaces. IEEE Transactions on Pattern Analysis and Machine Intelligence 31(4), 637-648 (2009)

3. Bertrand, G.: On critical kernels. Comptes Rendus de l'Académie des Sciences, Série Math. I(345), 363-367 (2007) 
4. Bertrand, G., Couprie, M.: Two-dimensional thinning algorithms based on critical kernels. Journal of Mathematical Imaging and Vision 31(1), 35-56 (2008)

5. Bertrand, G., Couprie, M.: Powerful Parallel and Symmetric 3D Thinning Schemes Based on Critical Kernels. Journal of Mathematical Imaging and Vision 48(1), 134148 (2014)

6. Manzanera, A., Bernard, T., Prêteux, F., Longuet, B.: n-dimensional skeletonization: a unified mathematical framework. Journal of Electronic Imaging 11(1), 25-37 (2002)

7. Lohou, C., Bertrand, G.: Two symmetrical thinning algorithms for 3D binary images. Pattern Recognition 40, 2301-2314 (2007)

8. Palágyi, K.: A 3D fully parallel surface-thinning algorithm. Theoretical Computer Science 406(1-2), 119-135 (2008)

9. Tsao, Y., Fu, K.: A parallel thinning algorithm for 3D pictures. CGIP 17(4), 315331 (1981)

10. Palágyi, K., Kuba, A.: A parallel 3D 12-subiteration thinning algorithm. Graphical Models and Image Processing 61(4), 199-221 (1999)

11. Lohou, C., Bertrand, G.: A 3D 6-subiteration curve thinning algorithm based on p-simple points. Discrete Applied Mathematics 151, 198-228 (2005)

12. Bertrand, G., Aktouf, Z.: A three-dimensional thinning algorithm using subfields. In: Vision Geometry III, vol. 2356, pp. 113-124. SPIE (1996)

13. Németh, G., Kardos, P., Palágyi, K.: Topology preserving 3D thinning algorithms using four and eight subfields. In: Campilho, A., Kamel, M. (eds.) ICIAR 2010. LNCS, vol. 6111, pp. 316-325. Springer, Heidelberg (2010)

14. Kovalevsky, V.: Finite topology as applied to image analysis. Computer Vision, Graphics and Image Processing 46, 141-161 (1989)

15. Kong, T.Y.: Topology-preserving deletion of 1's from 2-, 3- and 4-dimensional binary images. In: Ahronovitz, E., Fiorio, C. (eds.) DGCI 1997. LNCS, vol. 1347, pp. 3-18. Springer, Heidelberg (1997)

16. Bertrand, G.: New notions for discrete topology. In: Bertrand, G., Couprie, M., Perroton, L. (eds.) DGCI 1999. LNCS, vol. 1568, pp. 218-228. Springer, Heidelberg (1999)

17. Bertrand, G., Malandain, G.: A new characterization of three-dimensional simple points. Pattern Recognition Letters 15(2), 169-175 (1994)

18. Bertrand, G.: Simple points, topological numbers and geodesic neighborhoods in cubic grids. Pattern Recognition Letters 15, 1003-1011 (1994)

19. Saha, P., Chaudhuri, B., Chanda, B., Dutta Majumder, D.: Topology preservation in 3D digital space. Pattern Recognition 27, 295-300 (1994)

20. Kong, T.Y.: On topology preservation in 2-D and 3-D thinning. International Journal on Pattern Recognition and Artificial Intelligence 9, 813-844 (1995)

21. Whitehead, J.: Simplicial spaces, nuclei and $m$-groups. Proceedings of the London Mathematical Society 45(2), 243-327 (1939) 